Fecha de recepción: mayo 2021 Fecha de aceptación: junio 2021 Versión final: julio 2021

\section{Reflexiones en torno al fortalecimiento de la Investigación en el Diseño, a través de una Enseñanza Dialógica}

María Teresa Alejandra López Colín ${ }^{(1)}$ y

Gabriel Gómez Carmona ${ }^{(2)}$

\begin{abstract}
Resumen: Ante una realidad compleja, cambiante y contradictoria, las áreas del conocimiento están obligadas a dialogar transdisciplinariamente para construir respuestas y soluciones a las múltiples necesidades sociales, reinventarse y evolucionar, impactando positivamente la formación de nuevos cuadros de profesionales. Ante ello, el diseño requiere fortalecer la investigación en sus procesos de enseñanza para mejorar su práctica y consolidar su pertinencia social. En este escenario de diálogo disciplinar y transdisciplinar, surge un cuestionamiento ¿Cómo fortalecer la investigación desde la enseñanza del diseño? La postura abordada discute la necesidad de implementar acciones para fomentar la cultura de la investigación para una formación integral de los profesionistas del diseño, pues de no hacerlo, se corre el riesgo de que los futuros diseñadores no sean aptos para integrarse a equipos inter y transdisciplinarios de trabajo. Por tal motivo, el presente artículo reflexiona sobre la necesidad de una enseñanza del diseño integral, con base en el diálogo disciplinar que evidencia las fortalezas de consolidar a los docentes y estudiantes en una cultura de la investigación ante los desafíos del mundo actual.
\end{abstract}

Palabras claves: diseño, investigación, enseñanza dialógica, dialogo transdisciplinar.

[Resúmenes en inglés y portugués en la página 53]

(1) Diseñadora Gráfica, Maestra en Administración, Doctora en Educación por la Universidad de Ixtlahuaca CUI. Docente-investigadora del UICUI. Profesora de la Licenciatura en Diseño Gráfico de la Facultad de Arquitectura y Diseño de la Universidad Autónoma del Estado de México. Reside en Toluca, México.janyloc06@gmail.com teresa.lopez@uicui.edu.mx

(2) Arquitecto, Maestro en Antropología Social y Doctor en Urbanismo. Profesor-Investigador de la Facultad Mexicana de Arquitectura, Diseño y Comunicación (FAMADYC) de la Universidad La Salle, México. Miembro del Sistema Nacional de Investigadores (SNI), de CONACYT. gabriel.gomez@lasalle.mx 


\title{
Introducción
}

\author{
No hay enseñanza sin investigación, \\ ni investigación sin enseñanza. \\ Paulo Freire
}

Ante una realidad compleja, cambiante y contradictoria, la sociedad requiere de profesionistas capaces, comprometidos con la solución de problemáticas sociales y la generación de desarrollo desde el conocimiento científico, producto de un trabajo colaborativo que dialogue desde la inter y la transdisciplina. El momento histórico actual tiene como una de sus variables un contexto digital con una sociedad red donde la información está al alcance de la mayoría y en cantidades inimaginables, lo que exige una capacidad de búsqueda, selección, análisis, síntesis y sentido crítico, para discernir entre todas las fuentes de información al alcance. Ante este escenario, todas las áreas del conocimiento están obligadas a dialogar transdisciplinariamente para construir respuestas y soluciones a las múltiples necesidades sociales, reinventarse y evolucionar, impactando positivamente la formación de nuevos cuadros de profesionales y el diseño no es la excepción.

Además del desarrollo de las habilidades blandas, transversales o también llamadas socioemocionales, como: gestión de conflictos, manejo de estrés, gestión del tiempo, gestión del cambio, trabajo colaborativo y liderazgo para una mejor convivencia en un entorno diverso, una visión desde la multiculturalidad representa una valiosa herramienta para una sana relación en equipos interdisciplinarios de trabajo o para la interacción en comunidades virtuales transdisciplinarias, lo que permite inferir desde este contexto, que la enseñanza en el diseño requiere actualmente formar a sus nuevos cuadros de futuros profesionistas con las habilidades, conocimientos y actitudes que les permitan integrarse a diversas áreas con la posibilidad de desarrollar mejores propuestas de solución a las necesidades sociales a partir de una cultura consolidada en la investigación.

Frente a esta situación, al profesionista del diseño se le exige mantenerse como observador de su entorno, capaz de identificar las necesidades de su contexto, para desde el diseño, trasformar en áreas de oportunidad dichas necesidades, por lo que, no basta con tener conocimientos sobre discurso visual, generación de estrategias de comunicación visual, gestión del diseño, manejo de software, dominio de un segundo idioma o capacidad colaborativa para desenvolverse en el espacio laboral con disposición a la movilidad o al teletrabajo con equipos de trabajo. Hoy en día, más allá de todo eso es de suma importancia la capacidad de cuestionar, analizar, sintetizar, reflexionar y debatir, a partir de un pensamiento crítico y complejo, utilizando una argumentación que invite al diálogo y a la toma de conciencia desde distintos enfoques para generar posibles soluciones, con el convencimiento de que su quehacer impacte positivamente en la sociedad, lo que garantiza mantener vigente su profesión desde una visión integral (las partes y el todo), más allá de enfoques simplistas y fragmentados de la realidad.

Por lo anterior, la enseñanza y promoción de la investigación a lo largo del proceso de formación del profesionista del diseño, permite desarrollar en él capacidades como: observación, percepción, pensamiento lógico, reflexivo y crítico, análisis, síntesis, interpretación 
en construcción conceptual, metodológica y metacognitiva, y diálogo transdisciplinar, que le ayudarán a detectar y solucionar problemáticas reales en el ejercicio de su profesión. Sin embargo, encontramos que en la mayoría de los perfiles de egreso de las licenciaturas en Diseño Gráfico en México aún existe una tendencia a instruir en la capacidad de dominio de los procesos metodológicos, el análisis, la síntesis y la reflexión, para lograr tan sólo integración entre teoría y práctica de forma general.

No obstante, pocas universidades en el país como la Universidad Autónoma Metropolitana Unidad Xochimilco, plasman en su perfil de egreso la capacidad de investigación, razón por la cual, se debe enfatizar la investigación como parte esencial de las habilidades de los futuros profesionistas. La relevancia de la pesquisa se sustenta en que este campo en el ámbito del diseño es amplio, y la comunidad de investigadores busca, dentro de trabajos inter o transdisciplinarios, generar conocimiento o aportes que beneficien a la disciplina, si ésta no forma parte de sus actividades prioritarias, su propósito, manifestado en el perfil de egreso, se convierte en una intención sin soporte sólido, por lo que, la problemática comienza desde la propia construcción del propósito educativo (Rivera, 2018, p. 111).

Por otro lado, al interior de las universidades en los últimos años se han incorporado muy variadas modalidades de titulación que buscan aumentar la eficiencia terminal, por lo que, a pesar de que éstas apoyan a los egresados a concluir eficientemente sus licenciaturas y obtener un título universitario, en la mayoría de los casos se relega la elaboración o conclusión del trabajo de grado y por tal motivo, la sustentación de una tesis. Lo antedicho, genera que los estudiantes dejen de lado la importancia que tiene la investigación y el positivo impacto que una tesis tendrá en su futuro ámbito profesional.

Por ello, desde esta perspectiva, diversos autores han abordado la necesidad de promover la investigación en la formación de los profesionales del diseño, tal es el caso de Martínez de la Peña (2017) quien menciona que:

existen instituciones donde sí se entiende al diseñador como investigador nato que resuelve problemas; donde se reconoce el valor y la importancia de la investigación como una actividad rigurosa que están buscando muchos empleadores para diagnosticar y resolver problemas y, por tanto, están actuando en consecuencia. Si el alumno no aprende a investigar de manera rigurosa, o realiza estas actividades superficialmente, sólo se le está enseñando a simular. (p. 31)

Si bien es en los posgrados donde se halla un mayor interés por fortalecer la investigación, Margolin (2005) afirma que ningún programa de posgrado en diseño (de maestría o de doctorado), ha familiarizado a los estudiantes con las áreas de investigación en diseño. En consecuencia, los programas académicos de diseño proponen un tema de estudio restringido (p. 337). Sin embargo, hay que señalar que para el caso mexicano, la habilidad para desarrollar investigación es una las categorías de evaluación de los propios programas de diseño, por lo que es necesario fortalecerla desde la formación en la licenciatura (pregrado), lo que incluso debe desembocar en una formación docente continua bajo esta línea. 
La investigación tiene como fin la construcción de nuevos conocimientos. Es la función que distingue a las IES de otras instancias, como los centros de capacitación empresariales. En su accionar, la investigación desarrolla el espíritu crítico de profesores y estudiantes y con esto coopera en la formación de analistas simbólicos. A partir de un contexto amplio, COMAPROD reconoce que existen diversos tipos de investigación que van desde la básica hasta la aplicada. Así mismo, parte de la base de que la investigación educativa es fundamental para el enriquecimiento de los programas académicos. (COMAPROD, 2017, p. 08).

Desde este escenario, el presente artículo reflexiona sobre la necesidad de una enseñanza del diseño integral, con base en el diálogo disciplinar, que evidencie las fortalezas de consolidar a los docentes y estudiantes en una cultura de la investigación ante los desafíos del mundo actual. El artículo presenta así, a partir de un análisis teórico crítico, la pertinencia del fortalecimiento de la investigación en el diseño, a través de una enseñanza con enfoque dialógico.

\section{El fin de la Investigación}

La generación de nuevo conocimiento es el resultado de la investigación continua sobre los distintos aspectos en la experiencia humana, a partir de su realidad cercana. Lo antedicho, obliga a un cuestionamiento y diálogo continuo con la finalidad de dar respuesta. El diccionario de la RAE define el término investigar como: Realizar actividades intelectuales y experimentales de modo sistemático con el propósito de aumentar los conocimientos sobre una determinada materia. (RAE, 2006).

Así, se puede afirmar que la investigación es la base del desarrollo científico y tecnológico al generar conocimiento teórico de frontera o de aplicación práctica, y si el objetivo de las universidades es la formación de ciudadanos competentes para el desarrollo de su sociedad, dicho propósito se logra mediante dos funciones: la educación y la investigación. Es a partir de la segunda mitad del siglo XX, que se buscó en contextos como el europeo (Inglaterra) y el norteamericano, desarrollar — desde un enfoque más cientificista - la práctica al interior de las áreas del diseño. Algunos de los enfoques generados desde la década de 1960 han ido de la mano de autores como Broadbent, Jones, Archer o Alexander, quienes los exponen en el famoso Simposio de Portsmouth de 1967 (Broadbent, 1971). Estos se desarrollaron hasta casi dos décadas después (Margolin, 2005) y han buscado, desde la mirada de la ciencia, organizar el proceso de diseño, y por consiguiente, el acto creativo. Esta orientación estuvo estuvo enfocada hacia un proceso más racional susceptible de generar hipótesis sujetas a verificación y donde la fuerte carga subjetiva -integrada por las características sociales, culturales, afectivas, cognitivas y espirituales del diseñador-, debía controlarse, o bien, reducirse al máximo por inscribirse en lo no racional y lo subjetivo, por carecer de rigor metodológico y verificación. 
Estos enfoques concibieron al diseño como susceptible de una racionalización de corte científico, es decir, que podía ser trabajado con el rigor del método científico. Ello dio lugar a una serie de propuestas que pugnaron por un ordenamiento metodológico del diseño a través de un conjunto de pasos ordenados, lógicos y sistematizados que, más allá del proceso creativo mismo, permitieran al diseñador, llegar en el menor tiempo posible y con el menor costo, a más y mejores soluciones de un problema de diseño. Parte de sus premisas consistieron en:

- Hacer visible el proceso lógico seguido por el diseñador, para llegar a una solución concreta.

- Control del proceso de diseño limitando al máximo los errores.

- Entender el proceso de diseño como una actividad más racional, lógica y sistemática (el diseñador como "caja de cristal"); que intuitiva o puramente creativa (el diseñador como "caja negra" o el diseñador como "mago") (Broadbent, 1971).

Estas propuestas metodológicas constituyeron algunos de los primeros intentos por acercar la práctica del diseño con la ciencia y la investigación. Luego de ello, el diálogo entre el diseño y las disciplinas científicas fue cada vez más estrecho y buscó, ante todo, dotarlo de mayor rigor, pues solía considerarse al diseño como una disciplina sin una cultura de investigación convincente (Ariza, 2012). Sin embargo, no hay que olvidar que muchos de estos métodos de diseño se volvieron rígidos y condujeron a posturas polarizadas, razón por la cual, varios de sus autores, (Jones o Alexander) al cabo de poco más de una década abandonaron el movimiento y cuestionaron seriamente sus premisas (Margolin, 2005).

Vale recordar que el diseño es una forma particular de producir conocimiento, pero no puede considerarse una ciencia, porque la formulación del conocimiento científico busca las leyes que rigen el universo y el comportamiento de los individuos, a diferencia del objetivo del diseño que es la planeación, la invención y producción de cosas nuevas. En efecto, puede definirse al diseño como una disciplina, que en la cultura, tiene por fenómeno de estudio el mundo artificial y también una implementación de estrategias específicas para la solución de problemas (Ariza, 2012).

Desde esta perspectiva, el diseño se ha tenido que adecuar al tipo de investigación reconocido en otras áreas de estudio con mayor tradición científica, para poder construir un discurso que le permita una validación y, consecuentemente, un reconocimiento; además de encontrar un lenguaje general que le permita dialogar no sólo con otras disciplinas, sino con los receptores de su trabajo: las personas (Ariza, 2012).

Este aspecto es importante, pone en relieve la relevancia que ha tenido para el diseño poder entrar en un diálogo, no sólo disciplinar (desde su propia esencia y razón de ser), sino particularmente transdisciplinar, para no quedar a la zaga del discurso y práctica del resto de otras áreas del conocimiento o de disciplinas de corte científico. En efecto, de no hacerlo, su propia pertinencia hubiera quedado en entredicho al no poder comprender y responder a una realidad contemporánea compleja que requiere ese diálogo transdisciplinar. 
Diversos han sido los enfoques orientados a dar cuenta de los alcances, direcciones o las posibles líneas a desarrollar en la investigación del diseño. Tal es el caso de Margolin (2005), quien afirma que la investigación en diseño es una actividad con un alcance vasto, cuyos objetivos y métodos apenas comienzan a articularse. Otro ejemplo, es la propuesta de Christopher Frayling (citado por Margolin, 2005) quien propone tres modelos de investigación en diseño, que a su vez retoma del historiador y crítico de arte británico Herbert Read:

- Investigación en el arte y el diseño: incluye el tradicional terceto de historia, teoría y crítica, pero incorpora también la investigación estética o perceptual, así como la investigación de perspectivas técnicas, materiales y estructurales del arte y el diseño.

- Investigación a través del arte y el diseño: Se centra en los proyectos desarrollados en los talleres de diseño y se relaciona con lo que también se conoce en Gran Bretaña como investigación guiada por la práctica. Como ejemplos, cita la investigación del comportamiento de los materiales, la adaptación de una pieza de tecnología para realizar nuevas tareas o la documentación de un experimento práctico. En este tipo de investigación, la documentación de lo que se hace es un componente esencial.

- Investigación para el arte y el diseño: Según Frayling, la tercera categoría, es la más difícil de caracterizar. En esta área, la principal fuente de investigación es un objeto de arte o de diseño, o un conjunto de estos objetos (Margolin, 2005, pp. 352-353).

Hasta hoy en día, el diseño continúa siendo una actividad que busca la mejora continua del entorno del ser humano construyendo mensajes, estrategias, objetos y espacios para ofrecer una mejor calidad de vida en las áreas donde se implementarán dichos proyectos, a través de procesos de innovación que transformen e impacten la realidad. De este modo, el diseñador se convierte en un agente de cambio en su sociedad.

Por tal motivo, la formación de profesionales del diseño a nivel superior — con las características citadas - requiere de programas de estudio que enfaticen la necesidad de desarrollar investigación, al representar una capacidad que le otorgará distintas habilidades para consolidarse en el ámbito profesional. Nos encontramos, de este modo, ante el desafío de crear culturas académicas que fomenten en los estudiantes una comprensión más amplia de la investigación en diseño (Margolin, 2005, p. 337).

Es a partir de concebir a la investigación como un área fundamental al interior del diseño, lo que permite que ésta sea valorada por los estudiantes. Asimismo es deseable que se formen con la convicción de que su disciplina requiere de la investigación para ratificar su pertinencia social y generar procesos de evolución disciplinar (Ariza, 2012), en un diálogo continuo con otras áreas del diseño y, por extensión, con disciplinas afines y complementarias, lo que sin lugar a duda, promueve la inter y la transdisciplinariedad en la resolución de problemáticas sociales.

Actualmente, la sociedad demanda profesionistas que además del saber hacer disciplinario, desarrollen un pensamiento complejo que les permita comprender la realidad social de manera integral (el todo y las partes) sin reduccionismos, ni parcialidades de ésta, lo que los lleva a la indagación, consulta y trabajo transdisciplinario, atendiendo a la diver- 
sidad de las nuevas condiciones y necesidades sociales postpandemia, de la mano de la reflexión y el diálogo transdisciplinario.

Existen varias áreas de acción para el diseñador, sin embargo, más allá de su destreza con las técnicas o nuevas tecnologías para el desarrollo de sus creaciones, requiere ser un profesionista que se adapte a las nuevas condiciones y exigencias actuales, con una alta capacidad investigativa, por lo que es tarea esencial de la universidad formar este nuevo tipo de profesionales del diseño.

\section{Investigación en la enseñanza del diseño desde una perspectiva dialógica}

El diálogo es la acción de conversar exponiendo ideas de forma alternativa entre los interlocutores. Es mediante este proceso donde se genera una comunicación de las partes que lo establecen, de tal manera que se llega a un entendimiento mediante la argumentación. Así encontramos un modelo de enseñanza sustentado en la comunicación, propuesto por Paulo Freire (2005) quien establece que la naturaleza del ser humano es, de por sí, dialógica. El autor, postula que la comunicación tiene un papel fundamental en nuestras vidas, ya que estamos siempre comunicándonos con los demás, y es mediante el diálogo como llegamos a comprendernos y transformarnos (Freire, 2005).

La enseñanza desde la dialógica, la entenderemos entonces como una relación más profunda que una simple charla, debido a que se involucra en esta acción al interpretante, quien intenta formar la versión de un texto para hacerlo comprensible al otro, para expresar desde su razón. Así, el interpretante se convierte, al mismo tiempo, en un marco referente. En esta interacción se muestra, entonces, una dependencia mutua entre los actores al comunicarse, es así como el diálogo consiente participar lo que cada uno reflexiona de sí mismo con otros sujetos que comparten dicha reflexión, ya que solo se puede conversar sobre algo cuando se ha pensado en ello, dado que el pensamiento representa al objeto y el lenguaje al pensamiento. Por lo tanto, preguntarse por el mundo, por el medio, por la vida propia, tiene el propósito de saber quiénes somos y asemejar la relación con los demás, lo que va aconteciendo al tener el argumento de "los otros" (Lince, 2009). Es a través de la dialógica que se busca la reflexión y el cuestionamiento en el estudiante de diseño para que sea capaz de comprender y traducir, a partir de su interpretación, el entorno que lo rodea. Más aún, es el cuestionamiento constante el que le permite desarrollar su capacidad de diálogo mediante la argumentación fundamentada de su postura ante las diversas situaciones que se le presenten dentro de su quehacer como diseñador, más allá de solo construir su conocimiento, discutirlo con sus pares merced a la comunicación.

Así, el modelo educativo con enfoque dialógico favorece el desarrollo del pensamiento crítico-reflexivo generando la participación, a través del discurso para debatir, persuadir y reflexionar sobre diversas ideas. Esto coincide con las nuevas propuestas pedagógicas del siglo XXI, donde se subraya que el conocimiento no se piensa como la unión de proposiciones explicativas y conceptuales que el estudiante ha de asumir/adquirir por medio de la obtención de firmes competencias. Antes bien el conocimiento se propone como 
resultado de un diálogo permanente del estudiante y docente con sus oportunas transformaciones y con las socialmente instituidas (Carbonell, 2015).

El modelo educativo dialógico promueve, además, el cuestionamiento constante, de esta forma, el preguntar es, potencialmente, la destreza de pensar. Conseguimos expresar, interpretando el sentido de las palabras, de este modo, el preguntar y pensar son dos procesos intelectuales inherentes. Por un lado, quien pregunta establece la indagación reflexiva del conocimiento; y por el otro, si la persona piensa y tiene conciencia de ello, entonces es capaz de elaborar interrogaciones y posibles contestaciones; a partir de este ineludible vínculo se provocan nuevos conocimientos. El uso de la pregunta es sustancial porque propicia la reflexión, el planteamiento de problemas o hipótesis. Favorece, además la expresión oral y/o escrita, la comunicación entre estudiantes, su atención y la creación de un ambiente favorable de aprendizaje (Zuleta Araujo, 2005).

Rivera (2018), se pronuncia a favor de este modelo de educación dialógica para la enseñanza del diseño. El autor, expone la necesidad de consolidar a la Universidad como el espacio propicio para el diálogo y la reflexión, donde el pensamiento basado en el entendimiento sea trasmitido por las palabras a través de la discusión o el debate, con base en argumentos sólidos construidos desde los conocimientos de la disciplina. De este modo, la propuesta es entrelazar la teoría y la práctica con una argumentación dialógica, donde se discuta desde varias perspectivas por los estudiantes y el docente, la exposición de su punto de vista sobre el mismo tema, logrando una interacción que construya el conocimiento del estudiante sobre el diseño, bajo la guía del docente. Es gracias a este diálogo (profesor-estudiante) que se construye el aprendizaje (Rivera, 2021).

Más aún, una enseñanza dialógica se caracteriza por ser: colectiva, recíproca, apoyadora del alumno, acumulativa y propositiva. Por lo antedicho, se debe considerar construir en los estudiantes la capacidad crítica desde la selección de los contenidos de los materiales a utilizar. También se debe alentar la reflexión mediante la palabra, donde se descubra su propia realidad y se la exprese mediante el incesante cuestionamiento, más allá de respuestas preestablecidas. En este sentido, son las preguntas la base de una construcción mental, para dar explicaciones posibles a la realidad que se les presenta. Esta realidad se pretende entender al establecer relaciones que emergen ante el suceso y darle una respuesta mediante la racionalización, y no a través de una base de respuestas predeterminadas para ser memorizadas. La pregunta es problematizadora, en oposición de la pedagogía habitual (o también conocida "de respuesta") en que el catedrático aporta consigo una sucesión de contestaciones prefabricadas a cuestionamientos que también ya han sido creados, respuestas propensas a cancelar al sujeto estudiante y cercenar un acercamiento fundamental del conocimiento a partir del diálogo (Velazco y de González, 2008).

Es en este espacio de encuentro entre pares donde se construye un verdadero aprendizaje significativo de los futuros profesionistas del diseño y donde las instituciones deben ser conscientes del compromiso que esto conlleva. Si revisamos la responsabilidad de las universidades e Instituciones de Educación Superior (IES) para con la formación de nuevos profesionales del diseño, en México, se hallará que: 
Las funciones sustanciales de la institución universitaria son la docencia, la investigación, la difusión y la vinculación. Dichas funciones son interdependientes y tienen como fin la formación de analistas simbólicos; en otras palabras, la formación de personas que sean competentes en el ejercicio de su profesión, pero que también sean capaces de enriquecer, transformar y producir nuevas formas de su ejercicio profesional, con el fin de favorecer las condiciones de vida de su entorno. Las IES, pues, forman ciudadanos, y por lo tanto son organizaciones altamente complejas. (COMAPROD, 2017, p. 7).

Aquí, se da cuenta de que la investigación debe estar presente en las acciones que realizan las IES, como parte de la formación de los profesionistas del diseño. Si no se encuentra la investigación como propósito educativo, es decir, plasmado en el perfil de egreso como parte de las actividades principales del mismo, es un problema, pues le resta sustento a la disciplina. Tal como indica Rivera (2018), atender exclusivamente las necesidades del mercado, olvida integrar la investigación en los contenidos de los programas, por lo que los diseñadores, se quedan sin espacios para cultivar el pensamiento crítico que analice los fines y medios de la disciplina, a fin de formular nuevas maneras de ejercer la profesión, restando así a su carácter universitario.

\section{Dialogo entre investigación y enseñanza: el rol del docente en la formación de la cultura investigativa}

En este apartado se considera conveniente citar nuevamente a Rivera (2013), quien se ha ocupado de aportar al debate teórico sobre el diseño, valiosas contribuciones sobre su proceso formativo. Entre otros importantes puntos menciona, "de entrada, es inevitable y apremiante que la investigación, con los conocimientos, habilidades y destrezas que ésta conlleva, se arraigue en la cultura" (Rivera, 2013, p. 19). De este modo, señala la necesidad de transformar la práctica docente en la enseñanza del diseño, inclinándose por el modelo de educación dialógica y resaltando la necesidad de reforzar la investigación en la formación de los futuros profesionistas del diseño. Rivera (2013) subraya que la universidad es un espacio en donde el diálogo y la reflexión son indispensables, para arraigar una cultura investigativa entre sus actores.

A fin de lograr ese tipo de enseñanza en el diseño es necesaria una formación docente integral, que considere a la investigación como una habilidad que permita generar un pensamiento crítico en su práctica educativa. "Un profesor debe ser un profesional reflexivo, debe pensarse y auto respetarse como tal. Un profesor piensa antes que hacer, piensa después de hacer y piensa sobre cómo pensó" (Shön, citado por Rivera, 2021, p. 9).

Por esta razón, es fundamental que el docente sea consciente de fortalecer la investigación en la educación del diseño mediante la dialógica. Es menester que la formación de nuevos diseñadores, les admita moverse de un modelo tradicional a uno distinto como el modelo 
dialógico de la enseñanza, para que sean personas capaces de seleccionar la información desde un sentido crítico y repensar el propósito de su disciplina. Parte de estas nuevas concepciones asumen interdisciplina y el trabajo colaborativo, sumando la experiencia y el enfoque de otras disciplinas que lo lleven hasta la transdisciplina, para alcanzar una mejor perspectiva de una situación que lo ayude a descubrir la verdadera problemática, para que emerja, desde la dialógica, una solución. Dicha solución será una opción dentro de un sin número de posibilidades, eligiendo la más viable, la que se ajuste al momento y las condiciones del entorno, para así construir en conjunto con los distintos actores del proceso de la educación en diseño, tanto docentes, estudiantes e investigadores, un conocimiento que sea resultado del cuestionamiento, el diálogo y debate que aporte a la disciplina del diseño. Así:

La Investigación dialógica atañe por su parte a la situación en la que el conocimiento es co-construido por el profesor y los estudiantes mediante su colaboración en actividades conjuntas. Nótese así que la instrucción dialógica atañe a lo que hace el docente, en tanto que acá se trata de lo que hacen en conjunto, o colaborativamente, los estudiantes. (Velazco y de González, 2008, p. 465).

Por esta razón, se requiere integrar, desde las primeras etapas de la formación del estudiante, una cultura investigativa bajo el modelo de la dialógica y así, generar pequeñas comunidades de investigación entre estudiantes, docentes e investigadores desde la licenciatura. Lo antedicho, obliga a una formación docente continua que fortalezca en ellos el desarrollo de la investigación, bajo una dialógica que genera la relación lógica entre pregunta y respuesta por ser esta, la estructura de la experiencia y la base de la comprensión de las ciencias humanas. Interpretar algo es verlo como un texto, para concebirlo como respuesta a una pregunta antepuesta, apropiársela, para adoptarla luego como una pregunta propia, al convertirse en conocimiento a ser sociabilizado.

Un sujeto que no se interroga frente al mundo no tiene sentido, además de que las incógnitas sólo existen en un sujeto. Es a partir de esas incertidumbres que el conocimiento avanza y por tanto la forma de vida (Lince, 2009). Es la hermenéutica analógica una vía loable para afrontar la complicación de la interpretación desde la enseñanza del diseño. Rivera (2021), considera revalorar la figura del docente y que éste se convierta en un investigador. La educación superior tiene la responsabilidad de formar profesionistas que sean capaces de producir conocimientos sobre su disciplina para comprender la pertinencia social de su práctica, contribuyendo al desarrollo de la misma, así el docente es parte sustancial del proceso de enseñanza. Debe ser generador de experiencias de aprendizaje significativo, llevando la didáctica a los nuevos entornos digitales con el uso de los recursos tecnológicos como herramientas, además de ser incluyente ante la diversidad y la transdisciplina, y que transite por la complejidad para dejar la parcialidad de su disciplina. Por consiguiente, un profesor es obligatoriamente un investigador de su disciplina, no es un investigador que confiere un tiempo a la enseñanza, investigar es un aspecto que forma parte esencial de ser profesor. 


\section{Conclusiones}

Como quedó expuesto en las páginas anteriores, se requiere de una formación en la auténtica educación dialógica, mediante espacios de charlas para el enriquecimiento en la diversidad de pensamiento, donde se construyan alternativas y posibilidades de reflexionar desde la experiencia del otro. Al mismo tiempo, el docente debe ser un atento escucha que posibilite atender los diálogos entre los estudiantes e integrar discusiones a partir de la exposición de problemáticas. Un diálogo entre pares que a través del cuestionamiento, desafíe al estudiante para ejercer el análisis mediante su pensamiento lógico, en búsqueda de soluciones y a fin de organizar sus ideas para dar respuestas con argumentos sólidos y no quedarse de manera reducida y simplista, en respuestas ya preestablecidas.

Por tal motivo, es conveniente para este proceso, abrir programas de formación docente continua que ponderen desarrollar las competencias investigativas de los profesores y que promuevan, al mismo tiempo, la escritura en los diseñadores, ya que ésta refuerza establecer conceptualmente las prácticas, además de convertirse en algo necesario para crear redes de investigadores (Rivera, 2013). La escritura es otra de las habilidades que se han de fomentar y no dejarse a un lado en la enseñanza del diseño, no sólo el hacer mediante proyectos, sino el construir a través de argumentos válidos que demuestren la comprensión de las teorías y las problemáticas a las que se enfrentaron y cómo se dio la solución apropiada. El estudiante debe ser consciente de sus procesos de elaboración y a la vez, ser capaz de trasmitirlo de forma clara y coherente, tal como lo buscaron los diversos enfoques teórico-metodológicos del diseño.

Las habilidades de investigación del docente aportan en el aula, reflexión y discusiones que llevan a formar profesionistas con sentido crítico y capacidad de análisis consciente de sus decisiones, además de que favorecen la búsqueda de mejores y mayores aportaciones al conocimiento, a través del desarrollo de proyectos de investigación que contribuyan a la solución de problemáticas sociales. Lo antedicho lleva a buscar distintos espacios de difusión de las propuestas de colaboración con otros investigadores, profesores y estudiantes, en foros donde se dialogue y divulguen dichos trabajos para darle un sentido a la disciplina confirmando con ello, su pertinencia social.

Fortalecer al docente con las habilidades de investigación beneficia a los estudiantes, ya que se integran en la didáctica, aumentando el diálogo que invita a los estudiantes a establecer sus proyectos de diseño como consecuencia de la reflexión sensata y crítica sobre su efectiva necesidad y alcance en su ejecución. Visualizar el abanico de posibilidades que la profesión ofrece en beneficio de la sociedad, los hace conscientes de su rol como profesionistas sumados al compromiso de la formación de los futuros estudiantes. El docente de diseño deberá investigar y reflexionar de manera ininterrumpida sobre el estado de la cuestión desde la epistemología de su disciplina, mediante el debate y discusión, constituyéndose como un investigador experto estimulando el aprendizaje de otros (Rivera, 2021). El no fomentar la investigación, deja a los estudiantes con pocas herramientas para su óptimo desempeño en su futura práctica profesional, pues sólo solucionarán las proble- 
máticas desde la periferia y sin ahondar otras cuestiones medulares. Se requiere como lo menciona Rivera (2021), darle una estructura e integrar los programas y contenidos con las líneas de investigación desde donde los docentes e investigadores creen las nuevas formas de enseñar y construir su disciplina. La investigación en el diseño debe intensificar la percepción y reflexión que resultan esenciales en cualquier actividad de diseño productiva. De esta manera, podrá contribuir a la formación de profesionales más responsables y poner de relieve, el valor del diseño como un componente de la cultura cuyo estudio nos concierne a todos (Margolin, 2005).

Por ello, se debe integrar desde el propósito mismo del programa de estudio en diseño, a la investigación, a través de un perfil de egreso que la considere parte fundamental en la educación de los diseñadores, para que se sumen en cada una de las actividades de las distintas etapas de la formación del profesionista. Así, mediante la complejidad creciente, fortalecer la reflexión, el diálogo y la escritura, sin olvidar que la capacitación en las habilidades investigativas no sólo se ha de dirigir hacia la comunidad de investigadores, sino hacia los docentes, que están en las distintas áreas de las academias de diseño. El objetivo consiste en la preparación de futuros diseñadores que sean capaces de auto gestionar su aprendizaje de una forma más eficiente y con estrategias de aprendizaje fortalecidas con la reflexión y el sentido de ser profesionistas conscientes de las distintas problemáticas que se enfrentarán, a través de la incorporación de una enseñanza dialógica, el pensamiento complejo y la transdisciplinariedad. En el actual contexto que se vive, y el futuro escenario global postpandemia, una de las pocas cosas seguras será la reinvención de la educación a todos los niveles, de la mano del diálogo disciplinar y transdisciplinar, que permitirá el avance de la ciencia y la generación de nuevo conocimiento a través del cual, será posible hacer frente a los inesperados y complejos desafíos globales que se avecinen.

\section{Lista de Referencias Bibliográficas}

Ariza, S. (2012). Investigación, diseño y los estudios de posgrado en México. En Ariza, S. La investigación en diseño, una visión desde los posgrados en México. (pp. 09-78). México: UACJ.

Broadbent, G. (1971). Metodología del diseño arquitectónico. Barcelona: GG.

Carbonell, J. (2015). Pedagogías del siglo XXI, Alternativas para la innovación educativa. Barcelona: Octaedro.

COMAPROD. (2017). Instrumento de evaluación de programas presenciales. México.

Freire, P. (2005). Pedagogía del Oprimido. México: Siglo XXI.

Lince Campillo, R. (2009). El papel de la hermenéutica en el diálogo que establecemos con nosotros y con los otros. Estudios Políticos, 9(18), 11-35 Recuperado de: https://www. redalyc.org/articulo.oa? $\mathrm{id}=426439977008$

Margolin, V. (2005). Las políticas de lo artificial, Ensayos y estudios sobre Diseño. México: Editorial Designio. 
Martínez de la Peña, A. (2017). Paradojas y reajustes de un concepto en boga. En Tapia, A., Esqueda, R., Lope- León, R., Martínez de la Peña, A., Rivera, A., Rodríguez L., Tiburcio, C., Torres, R., Villalobos, S. ¿Design Thinking? Una discusión a nueve voces, (pp. 29-33). México: Ars Optika.

RAE (2006). Diccionario esencial de la lengua española. Recuperado de https://www.rae.es/ obras-academicas/diccionarios/diccionario-esencial-2006

Rivera, A. (2013). La nueva educación del diseñador gráfico. México: Designo.

Rivera, A. (2018). La Evaluación de la educación del diseño en México: un enfoque desde la didáctica. México: COMAPROD.

Rivera, A. (2021). La precarización de la educación superior del Diseño Gráfico Entretejidos. Revista de transdisciplina y Cultura digital. Año 8, 1(14). Recuperado de https:// entretejidos.iconos.edu. $\mathrm{mx} /$ thesite/la-precarizacion-de-la-educacion-superior-deldiseno-grafico-notas-en-torno-a-una-reinvencion-pedagogica-borrador/

Tapia, A., Esqueda, R., Lope- León, R., Martínez de la Peña, A., Rivera, A., Rodríguez L., Tiburcio, C., Torres, R., Villalobos, S. (2017). ¿Design Thinking? Una discusión a nueve voces. México: Ars Optika.

Velasco, J.A. y de González, L. (2008). Sobre la teoría de la educación dialógica. Educere, 12(42), 461-470. Recuperado de https://www.redalyc.org/articulo.oa?id=35614569006

Zuleta Araújo, O. (2005). La pedagogía de la pregunta. Una contribución para el aprendizaje. Educere, 9(28), 115-119. Recuperado de https://www.redalyc.org/articulo. oa?id=35602822

\begin{abstract}
Faced with a complex, changing and contradictory reality, the areas of knowledge are obligated to dialogue transdisciplinary to build responses and solutions to multiple social needs, reinvent themselves and evolve, positively impacting the training of new professional cadres. Given this, design requires to strength the research in its teaching processes to improve its practice and consolidate its social relevance. In this scenario of disciplinary and transdisciplinary dialogue, a question arises: How to strengthen research from the teaching of design? The position addressed discusses the need to implement actions to promote the culture of research for a comprehensive training of design professionals, because if not, there is a risk that future designers will not be able to join inter and transdisciplinary teams of job. For this reason, this article reflects on the need for an integral design teaching, based on the disciplinary dialogue that shows the strengths of consolidating teachers and students in a culture of research in the face of the challenges of today's world.
\end{abstract}

Keywords: design, research, dialogic teaching, transdisciplinary dialogue. 
Resumo: Perante uma realidade complexa, mutante e contraditória, as áreas do conhecimento são obrigadas a dialogar transdisciplinarmente para construir respostas e soluções às múltiplas necessidades sociais, reinventar-se e evoluir, impactando positivamente na formação de novos quadros de profissionais. Diante disso, o Design requer o fortalecimento da pesquisa em seus processos de ensino para aprimorar sua prática e consolidar sua relevância social. Nesse cenário de diálogo disciplinar e transdisciplinar, surge o questionamento: como fortalecer a pesquisa a partir do ensino do design? O posicionamento abordado discute a necessidade de implementar ações de fomento à cultura da pesquisa para a formação integral dos profissionais de design, pois, se não o fizer, corre-se o risco de os futuros designers não conseguirem integrar equipes de trabalho inter e transdisciplinares. Por isso, este artigo reflete sobre a necessidade de um ensino integral do design, a partir de um diálogo disciplinar que mostre os pontos fortes da consolidação de professores e alunos em uma cultura de pesquisa diante dos desafios do mundo atual.

Palavras chave: design, pesquisa, ensino dialógico, diálogo transdisciplinar.

[Las traducciones de los abstracts fueron supervisadas por el autor de cada artículo] 